\title{
Suprachoroidal Delivery of Viral and Nonviral Gene Therapy for Retinal Diseases
}

\author{
Viral Kansara, Leroy Muya, Chen-rei Wan, and Thomas A. Ciulla
}

\begin{abstract}
Retinal gene therapy is a rapidly growing field with numerous clinical trials underway, and route of delivery is a critical contributor to its success. Subretinal administration, which involves pars plana vitrectomy in the operating room, offers targeted delivery to retinal-pigment epithelium cells and photoreceptors. Due to the immune-privileged nature of the subretinal space, the risk of an immune reaction against viral capsid antigens is minimized, an advantage of subretinal administration in patients with preexisting neutralizing antibodies. Intravitreal administration, with fewer procedure-related complications, is challenged by potential immune response and incomplete vector penetration through the internal limiting membrane. However, novel vectors, optimized by "directed evolution" may address these issues. Nonsurgical in-office suprachoroidal gene delivery offers the potential for greater surface-area coverage of the posterior segment compared to focal subretinal injection, and is not hindered by the internal limiting membrane. However, the vector must pass through multiple layers to reach the targeted retinal layers, and there is a risk of immune response. This review highlights recent developments, challenges, and future opportunities associated with viral and nonviral suprachoroidal gene delivery for the treatment of chorioretinal diseases. While ocular tolerability and short-term effectiveness of suprachoroidal gene delivery have been demonstrated in preclinical models, durability of gene expression, long-term safety, potential systemic exposure, and effective delivery to the macula require further exploration. Although the safety and efficacy of suprachoroidal gene delivery are yet to be proven in clinical trials, further optimization could facilitate nonsurgical in-office suprachoroidal gene therapy.
\end{abstract}

Keywords: suprachoroidal, gene therapy, viral vectors, nanoparticles, chorioretinal diseases

\section{Retinal Gene Therapy}

W ITH THE ADVANCEMENT in gene delivery technologies, recent innovations in genetic analysis for identifying monogenic disorders, and accessibility to local ocular delivery, the eye is a prime target for ocular gene therapy research. ${ }^{1}$

The eye offers a unique opportunity to monitor disease progression or therapeutic response through advanced noninvasive diagnostic technology. Due to its small size, and ability to deliver gene therapy directly into the eye, the need for total amount of genetic payload is low. Moreover, the confined anatomy of eye restricts the systemic exposure of administered vectors or nonviral nanoparticles to a minimum, while the relative immune-privilege nature of retina limits inflammatory response. However, the presence of various static and dynamic barriers, such as the internal limiting membrane as an inner retinal barrier, ${ }^{2}$ and the retinal-pigment epithelium (RPE)/Bruch's membrane complex as an outer blood retinal barrier, complicates delivery of gene therapy to the retina.

Nevertheless, the intense focus on gene therapy for ocular diseases resulted in the first Food and Drug Administration (FDA)-approved gene therapy in 2017 for patients with RPE65 mutation-associated inherited retinal dystrophy. ${ }^{3}$ This approval paves the way for a new wave of innovation in retinal gene therapies.

The growing field of retinal gene therapy encompasses the treatment of inherited retinal diseases (IRDs), as well as treatment of noninherited chronic disorders. For the treatment of autosomal recessive IRDs, such as retinitis pigmentosa (RP), Leber congenital amaurosis (LCA), X-linked

Clearside Biomedical, Inc., Alpharetta, Georgia.

(C) Viral Kansara et al. 2020; Published by Mary Ann Liebert, Inc. This Open Access article is distributed under the terms of the Creative Commons Attribution Noncommercial License (http://creativecommons.org/licenses/by-nc/4.0/) which permits any noncommercial use, distribution, and reproduction in any medium, provided the original author(s) and the source are cited. 
retinoschisis, achromatopsia, Usher syndrome, and Stargardt disease, gene augmentation is a potentially beneficial strategy. ${ }^{4}$ The disease-causing genetic mutation leads to total absence or near absence of functional protein in retina. Gene augmentation in these cases can correct the loss of function by delivering normal copies of the functional gene; in some instances, restoring a small percentage of the gene product can revert the phenotype. Several gene therapy-based clinical trials are underway for the treatment of IRDs. ${ }^{5}$ For the treatment of noninherited chorioretinal disorders, such as age-related macular degeneration, diabetic retinopathy, diabetic macular edema, geographic atrophy, and uveitis, clinical trials are currently assessing gene therapy with transgenes encoding therapeutic proteins. ${ }^{6}$ This approach has been utilized to express antiangiogenic therapeutic proteins, such as pigment epithelium-derived factor (PEDF), ${ }^{7,8}$ soluble fms-like tyrosine kinase-1 (sFLT-1), ${ }^{9}$ angiostatin- and endostatin-like proteins, ${ }^{10}$ as well as aflibercept-like ${ }^{11}$ and ranibizumab-like proteins. ${ }^{12}$ Inhibition of components of the complement cascade through gene therapy approach is also currently under investigation. ${ }^{13}$

The purpose of this review article is to discuss three key routes of retinal gene delivery-subretinal, intravitreal, and suprachoroidal administration-with a specific focus on recent advances, challenges, and future opportunities associated with suprachoroidal gene delivery for posterior segment diseases.

\section{Routes of Administration for Retinal Gene Delivery}

Subretinal, intravitreal, and suprachoroidal administration are the three routes of administration for retinal gene therapy (Fig. 1). Delivery of viral vectors and nonviral nanoparticle-based gene therapy by each of these routes offers unique advantages and faces specific challenges.

\section{Subretinal administration}

Subretinal administration is the most investigated method for retinal gene therapy and involves pars plana vitrectomy (PPV) followed by a "retinotomy," to facilitate subretinal administration of the viral vectors. This method allows for direct delivery of gene therapy into the subretinal space, a virtual space between the RPE cells and photoreceptors (PRs), and hence offers targeted delivery to outer retinal cells, including RPE cells and PRs. Due to the immune-privilege nature of the subretinal space, subretinally-injected adeno-associated viruses (AAVs) may not be affected by preexisting neutralizing antibodies (NAbs) against AAV, and consequently, this route may minimize the risk of an immune reaction against viral capsid antigens. ${ }^{5}$ Thus, subretinal injection has become the most commonly utilized route of administration. Several clinical trials have been completed or are currently in progress for inherited and non-IRDs. ${ }^{14}$

Subretinal delivery, however, is an invasive route of administration as it causes a temporary focal retinal detachment and creation of a retinotomy, and therefore can pose higher risks for patients whose retinal cellular integrity has already been compromised. Moreover, the vitrectomy procedure, which facilitates subretinal administration, carries a high risks of inducing cataracts and a low risk of retinal detach-

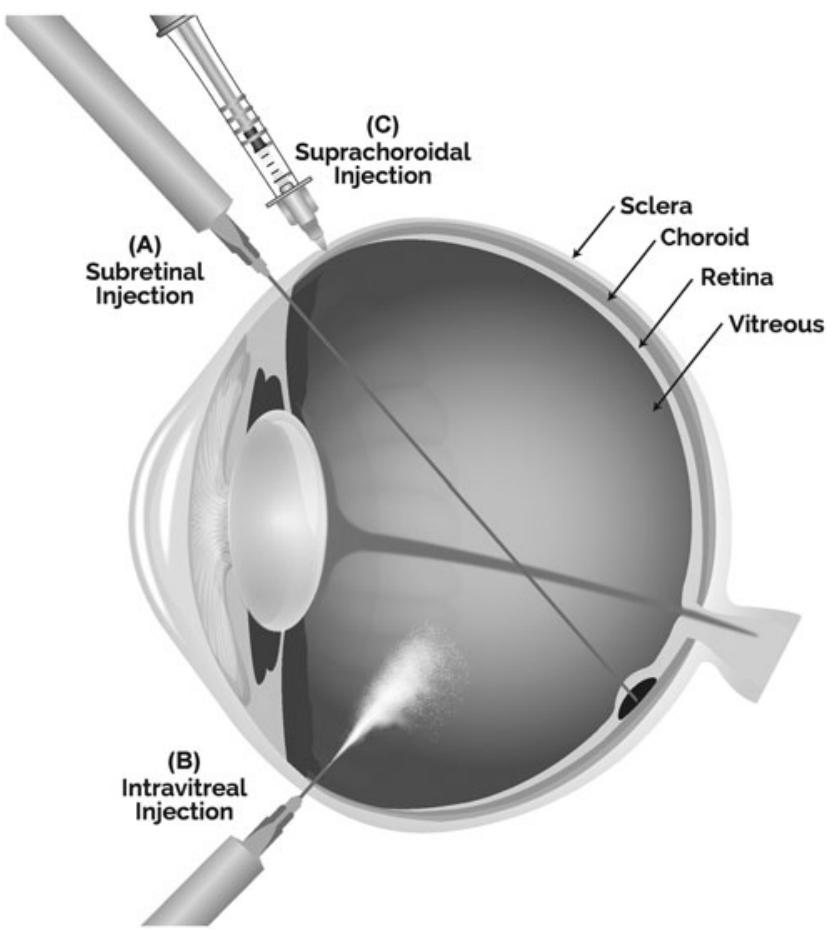

FIG. 1. Routes of administration for retinal gene delivery (A) subretinal, (B) intravitreal, and (C) suprachoroidal through a microneedle. Subretinal injection, performed in the operating room, delivers vectors focally to the subretinal space, a virtual space between the retinal pigment epithelial cells and photoreceptors. Intravitreal injection, performed in an office setting, delivers vectors to the vitreous humor. Suprachoroidal injection by a microneedle, nonsurgical inoffice procedure, delivers vectors to the suprachoroidal space (SCS), a virtual space between choroid and sclera. Once administered in the SCS, the injectate spreads posteriorly and circumferentially.

ment. Another limitation of the subretinal delivery approach is that the spread of the delivered vectors to the subretinal space is minimal, mostly limited to the area near the injection site. Therefore, subretinal delivery may result in suboptimal therapeutic benefits for diseases that benefit most from diffuse transduction of peripheral retina, such as RP.

\section{Intravitreal administration}

Intravitreal delivery is another promising administration route for retinal gene therapy. This method, compared to subretinal surgery, is less invasive and potentially has fewer procedure-related complications. Intravitreal gene therapy delivery may be most applicable to inner retina diseases, such as Leber hereditary optic neuropathy, and glaucoma, where transduction of the ganglion cell layer may provide therapeutic benefits. Intravitreal delivery may also facilitate in-office gene therapy with transgene encoding secreted therapeutic proteins, which do not rely on transduction of specific retinal cells.

Historically, the clinical success of intravitreal gene therapy for the treatment of IRDs has been limited ${ }^{15,16}$ due to the presence of the internal limiting membrane, which lines the inner retina, and can hinder the penetration of viral vectors to underlying retinal layers such as PR and RPE. In addition, intravitreal administration leads to the dilution of the therapeutic agent within the vitreous cavity. Further, 
unlike the subretinal space, NAbs against AAVs can access the vitreous, which may induce humoral immune response, diminish therapeutic response, and increase safety risks. ${ }^{17}$ These potential limitations of intravitreal gene delivery may be addressed by techniques such as "directed evolution" and "rational design" to generate "designer" vectors. ${ }^{18}$ For example, vectors have been developed in which capsid tyrosines were mutated to phenylalanines, which decrease the risk of immune response and can increase transgene expression. ${ }^{19}$

\section{Suprachoroidal administration}

Recently, drug delivery through the suprachoroidal space (SCS), the potential space between the sclera and the choroid, has undergone successful phase 3 clinical study. ${ }^{20}$ Access to the SCS can be achieved by three main methodscatheters, needles, and microneedles. Catheter-based technology, such as the iTrack ${ }^{\mathrm{TM}} 250 \mathrm{~A}$ microcatheter, is performed by inserting a catheter through a small sclerotomy incision into the SCS and advancing the catheter posteriorly toward the optic disc. ${ }^{21}$ The catheter may be illuminated at the tip and thus can be visualized intraoperatively. This can facilitate delivery to specific regions of the retina, including the macula. The drawback to the catheter-based procedures is the relative invasiveness, generally requiring an operation room setting.

Another method to access the SCS is the free-hand technique using a standard hypodermic needle attached to a Hamilton syringe or insulin syringe. ${ }^{22}$ In this method, the needle is inserted directly and tangentially through the sclera behind the limbus with or without sclerotomy. The needle is then slowly advanced with gentle pressure on the plunger, and the injection slowly performed when the loss of resistance is experienced. The advantage of using standard hypodermic needles is that the needles and syringes are readily available. However, the insertion depth and angle are difficult to control, thus increasing the propensity for inadvertent intravitreal or subretinal injections.

Advancements in microneedle technology have enabled nonsurgical in-office access to the SCS. Microneedle technology, such as the SCS Microinjector ${ }^{\mathrm{TM}}$, has been evaluated both preclinically and clinically. ${ }^{20,23-26}$ Preclinical studies have demonstrated targeted and compartmentalized drug delivery to the sclera-RPE-choroid and retina following microneedle-based suprachoroidal administration. Microneedle technology allows for precise control in reaching the SCS, compared to standard hypodermic needles, as short needles (typically, 900 and 1,100 $\mu \mathrm{m}$ ) guide and limit penetration to the SCS. Needle lengths at this range penetrate the sclera to reproducibly access and deliver drug to the SCS. Once in the SCS, the injectate spreads posteriorly and circumferentially. Unlike the catheter-based procedure, suprachoroidal injections with microneedles can be performed in an office setting under aseptic conditions without sclerotomy.

Suprachoroidal gene delivery may offer potential advantages over intravitreal delivery or even the subretinal route. Unlike subretinal delivery, suprachoroidal delivery does not require retrobulbar anesthesia in an operating room; PPV with associated complications of cataract, retinal tear, or retinal detachment; creation of a retinotomy, which can be complicated by hemorrhage; or creation of a bleb, which can be complicated by central vision loss from foveal atrophy or macular hole. ${ }^{27}$ Moreover, suprachoroidal delivery offers the potential for greater surface-area coverage of the posterior segment compared to focal subretinal administration. Unlike intravitreal administration, suprachoroidal delivery is not hindered by the internal limiting membrane or the potential for particles/floaters in the visual axis.

Suprachoroidal gene therapy delivery, however, may face several challenges. Effective transduction of retina after suprachoroidal administration could be hindered due to the rapid clearance by the choriocapillaris, although the choroidal vascular pore size may limit entry of vectors or nonviral nanoparticles. ${ }^{28}$ Although limited systemic absorption is expected after suprachoroidal delivery, the possibility of systemic exposure cannot be completely ruled out. Therefore, thorough assessment of gene transfer to nonocular tissues is warranted. Another potential challenge is the lack of immune privilege due to anatomical location of the SCS, outside the blood-retinal barrier. Therefore, the impact of preexisting NAbs against the virus needs to be carefully considered for the suprachoroidal gene therapy.

\section{Suprachoroidal Gene Delivery}

A variety of viral and nonviral nanoparticles have undergone preclinical assessments for suprachoroidal delivery over the past two decades (Table 1).

\section{Suprachoroidal viral vector-based retinal gene delivery}

AAVs are the most studied viral vectors in the field of suprachoroidal gene therapy due to their history of safety and efficacy in human clinical trials. Feasibility of suprachoroidal gene delivery through a microcatheter was evaluated almost a decade ago with the goal to develop a less invasive delivery method, compared to a standard 3-port PPV/subretinal injection. Peden et al. assessed ocular tolerability and gene transfer ability of AAV5 delivered to the SCS using a catheter-based system in rabbits. This delivery procedure was found to be tolerable, and showed no evidence of retinal detachment, ocular infection, vitreous hemorrhage, or other adverse events. Six weeks after surgery, immunostaining demonstrated robust transduction of green fluorescent protein (GFP) in all treated eyes, with transfection occurring at the level of the choroid, RPE, PRs, and retinal ganglion cells. The authors concluded that this microcatheter approach was successful in safely and effectively delivering a gene therapy agent to the SCS, representing a less invasive alternative to PPV with subretinal injection. ${ }^{21}$

Growing understanding of AAV biology has led to the design and development of recombinant AAV serotypes that may result in improved gene transfer efficiency, and cell specificity, that is, tropism. Martorana et al. compared transduction efficiency of three different AAV serotypes after suprachoroidal administration by an iTrack illuminated microcatheter (iScience, CA) in New Zealand White rabbits. $^{29}$ These serotypes included AAV serotype 2 (AAV2), AAV5, and AAV2 containing 3 tyrosine-phenylalanine mutations on the capsid surface [AAV2(triple)]. Efficiency of suprachoroidal and subretinal gene transfer was further compared in rabbits. GFP expression was observed in all eyes that received vitrectomy/subretinal or suprachoroidal injections, with AAV2 producing the strongest GFP 
Table 1. Summary of Preclinical Studies of Suprachoroidal Gene Delivery

\begin{tabular}{|c|c|c|c|c|c|c|}
\hline Technology & Gene & Species & $\begin{array}{c}\text { Disease } \\
\text { model }\end{array}$ & Duration & Key outcome & References \\
\hline \multicolumn{7}{|c|}{ Viral-based gene therapy } \\
\hline AAV2 & GFP & Mouse & None & 6 weeks & $\begin{array}{l}\text { GFP expression observed } \\
\text { in RPE and multiple } \\
\text { retinal layers }\end{array}$ & 30 \\
\hline $\begin{array}{l}\text { AAV2, } \\
\text { AAV5, } \\
\text { AAV2(triple) }\end{array}$ & GFP & Rabbit & None & 4-6 weeks & $\begin{array}{l}\text { GFP expression was } \\
\text { observed for all } 3 \\
\text { serotypes. AAV2 was } \\
\text { the strongest }\end{array}$ & 21,29 \\
\hline $\begin{array}{l}\text { AAV2, } \\
\text { AAV8, } \\
\text { AAV9 }\end{array}$ & GFP & Rat & None & $\begin{array}{l}\text { Up to } \\
2 \text { weeks }\end{array}$ & $\begin{array}{l}\text { GFP expression observed } \\
\text { in the RPE and } \\
\text { photoreceptors } \\
\text { AAV2 did not show } \\
\text { expressions as strongly } \\
\text { as AAV8 and AAV9 }\end{array}$ & 22 \\
\hline AAV8 & $\begin{array}{l}\text { Anti-VEGF } \\
\text { Fab }\end{array}$ & Rat & $\begin{array}{l}\text { VEGF- } \\
\text { induced } \\
\text { retinal } \\
\text { vascular } \\
\text { leakage }\end{array}$ & $\begin{array}{l}\text { Up to } \\
7 \text { weeks }\end{array}$ & $\begin{array}{l}\text { Reduced vessel dilation } \\
\text { and leakiness } \\
\text { SC and SR injections } \\
\text { produced similar levels } \\
\text { of anti-VEGF Fab } \\
\text { protein levels between } \\
2 \text { and } 7 \text { weeks }\end{array}$ & \\
\hline AAV8 & GFP & $\begin{array}{l}\text { Monkey, } \\
\text { Yorkshire } \\
\text { pig }\end{array}$ & None & 21 days & $\begin{array}{l}\text { GFP expression observed } \\
\text { in the RPE } \\
\text { Generally tolerated }\end{array}$ & \\
\hline AAV8 & GFP & Monkey & None & $\begin{array}{l}1-3 \\
\text { months }\end{array}$ & $\begin{array}{l}\text { Diffuse peripheral GFP } \\
\text { expression in RPE } \\
\text { Infiltration of } \\
\text { inflammatory cells } \\
\text { observed }\end{array}$ & 31 \\
\hline \multicolumn{2}{|c|}{$\begin{array}{l}\text { Non-viral gene therapy } \\
\text { Electrotransfer }\end{array}$} & Rat & $\mathrm{CNV}$ & 15 days & $\begin{array}{l}\text { Inhibition of choroidal } \\
\text { neovascularization } \\
\text { observed in flat mount }\end{array}$ & 34 \\
\hline & $\beta$-galactosidase & & None & $\begin{array}{l}\text { Up to } \\
4.5 \text { months }\end{array}$ & $\begin{array}{l}\text { Gene expression was most } \\
\text { prominent on day } 7 \text { and } \\
\text { decreases over time }\end{array}$ & \\
\hline Liposome & TIMP-2 & $\begin{array}{l}\text { Guinea } \\
\text { pig }\end{array}$ & Myopia & 14 days & $\begin{array}{l}\text { Changes to collagen I and } \\
\text { fibronectin mRNA } \\
\text { expressions }\end{array}$ & 39 \\
\hline \multirow[t]{2}{*}{ DNPs } & Luciferase & Rabbit & None & 7 days & $\begin{array}{l}\text { Luciferase activity } \\
\text { observed in retina and } \\
\text { RPE/choroid } \\
\text { Suprachoroidal and } \\
\text { subretinal injection } \\
\text { produced comparable } \\
\text { luciferase activity }\end{array}$ & 48,49 \\
\hline & & NHP & None & 21 days & $\begin{array}{l}\text { Luciferase activity } \\
\text { observed in retina and } \\
\text { RPE/choroid }\end{array}$ & \\
\hline
\end{tabular}

AAV, adeno-associated virus; AAV(triple), AAV2 containing 3 tyrosine-phenylalanine mutations on the capsid surface; CNV, choroidal neovascularization; DNPs, DNA nanoparticles; GFP, green fluorescent protein; NHP, nonhuman primate; RPE, retinal-pigment epithelium; SC, suprachoroidal; sFlt-1, soluble fms-like tyrosine kinase-1; SR, subretinal; TIMP-2, tissue inhibitor of metalloproteinases 2; VEGF, vascular endothelial growth factor.

expression. More importantly, the authors concluded that this ab-externo suprachoroidal approach mediates AAV transduction of the posterior retina, similar to that seen with PPV/subretinal injection.

Woodard et al. introduced AAV2 into the mouse eye through various routes, including suprachoroidal delivery. ${ }^{30}$ In this mouse model, AAV2 was used to deliver a selfcomplementary cytomegalovirus (CMV) promoter/GFP re- porter cassette. The authors observed expression of GFP in the retina at 6 weeks. Specifically, transduction in the RPE and multiple retinal layers was observed after suprachoroidal delivery. A confounding factor for this study could be the inadequate reliability and precision of suprachoroidal injections using a free-hand method in mouse eyes, which may affect the transduction and distribution pattern. Nonetheless, the ability to transduce retinal layers without 
inducing a temporary retinal detachment led the authors to conclude that suprachoroidal delivery may offer unique advantages over subretinal delivery.

Recently, transduction efficiency, durability, and pharmacological efficacy of suprachoroidal gene delivery have been assessed in rats, pigs, and nonhuman primates (NHPs) using a conventional hypodermic needle and a free-hand method. ${ }^{22}$ Ding et al. investigated suprachoroidal injection of AAV8 for ocular gene transfer using a GFP reporter gene, as well as RGX-314, a clinical grade AAV8 vector containing a gene cassette encoding a humanized monoclonal antigen-binding fragment that neutralizes human vascular endothelial growth factor (VEGF). RGX-314 utilizes a CB7 promoter consisting of the chicken $\beta$-actin promoter and CMV enhancer, a chicken $\beta$ - actin intron, and a rabbit $\beta$-globin polyadenylation signal. Suprachoroidal injection of AAV8.GFP in rats, NHPs, and pigs resulted in GFP expression in RPE and PRs throughout a large portion of the posterior segment. Interestingly, the retinal surface-area of GFP expression was augmented by a second suprachoroidal AAV8.GFP injection. This finding raises a possibility of using multiple suprachoroidal injections, in different quadrants of the eye, to improve the levels and area of expression throughout RPE-choroid, and retina.

The same investigators also compared the transduction efficiency of three different AAV serotypes-AAV2, AAV8, and AAV9-after suprachoroidal injections. Suprachoroidal AAV8 and AAV9, but not AAV2, encoded with GFP, elicited strong fluorescent signal in both the RPE and the PRs in rats. In addition, suprachoroidal and subretinal administrations of the AAV8 encoding ranibizumab-like anti-VEGF agent resulted in similar levels of anti-VEGF therapeutic protein and similar suppression of VEGF-induced vascular leakage in a rat model. No overt sign of ocular inflammation or immune response was observed after suprachoroidal AAV8. However, three rhesus monkeys in this study showed no detectable anti-AAV8 Nab before suprachoroidal injection, and consequently, the potential for suprachoroidal delivery in $\mathrm{Nab}+$ animals or patients is unclear. Nonetheless, suprachoroidal AAV-based gene therapy seems to be a promising therapeutic option that could lead to a noninvasive outpatient procedure for the treatment of retinal diseases.

More recently, microneedle-based suprachoroidal gene delivery was investigated in NHPs. ${ }^{31}$ In this study, Yiu et al. compared GFP expression among suprachoroidal, subretinal, and intravitreal gene delivery of AAV8 containing an expression cassette encoded for enhanced GFP under a ubiquitous CMV promoter, woodchuck hepatitis virus post-transcriptional regulatory element, and bovine growth hormone polyadenylation signal. Suprachoroidal injection of AAV8-eGFP resulted in widespread peripheral and circumferential transgene expression in RPE, while subretinal AAV8 produced focal transduction in the RPE, PRs, and some ganglion cells, and intravitreal AAV8 resulted in GFP expression in cells, possibly astrocytes, or Müller glia, in peripapillary region. The transduction of RPE and PRs after suprachoroidal and subretinal AAV8 delivery was found to be more efficient compared with intravitreal injections.

While Yiu et al. reported lower systemic humoral immune response with suprachoroidal AAV8, compared to intravitreal AAV8, intraocular inflammation was more evi- dent with AAV8 delivered to the SCS, compared to the subretinal space. This observation is not completely unexpected since the SCS, unlike the subretinal space, is outside the outer blood-retinal barrier, and hence, suprachoroidal AAV8 can be exposed to the local and systemic immune surveillance cells, such as macrophages. It is noteworthy that in this study, authors intentionally avoided the use of extensive immunosuppression, to understand the native immune response to suprachoroidal AAV8 delivery. For future studies, the use of topical or systemic corticosteroid is warranted. These authors also reported decreased transgene expression at the 2- and 3-month time points, compared to the 1-month time point, after suprachoroidal administration. This could be due to the phagocytic activity of infiltrated macrophages and leukocytes that was coincidentally observed at 1-month. These inflammatory cells would phagocytose transfected cells, and degrade them as a part of inflammatory response, which may have resulted in loss of gene expression after 1-month.

The results obtained from Ding et al. seem more favorable that those from Yiu et al. However, it is critical to note that gene therapies with the same or similar vector capsid can vary in multiple important ways, including transgene, codon optimization, regulatory elements, presence of residual empty capsids, purity of final formulation, and dose level. The small sample size, the use of NHP grade formulation, differences in transgene and regulatory elements, as well as lack of immunosuppression may have contributed to differences in outcomes, observed by Yiu et al. Several optimization approaches, such as the use of additional AAV serotypes, or AAV variants identified by in vivo-directed evolution, use of different promoters and enhancers, use of formulations prepared using good manufacturing practices, dose optimization, as well as employment of immunosuppression should be considered in future studies.

\section{Suprachoroidal nonviral gene delivery}

The application of nanoparticles for retinal gene delivery has attracted interest because of the potential to transfer large genes of common IRDs, such as Stargardt or Usher Syndrome, which are beyond the carrying capacity of AAVs. ${ }^{32}$ Moreover, the nonviral approach incurs less risk of immune response, especially since many patients have preexisting NAbs to AAV capsid antigens. ${ }^{33}$ Consequently, nonviral gene delivery offers the potential for titratable and repeatable dosing to accommodate and optimize clinical need.

One key limitation of nonviral gene therapy is the nuclear entry of the payload after entering the cells. Several methods, physical and chemical, have been assessed preclinically by suprachoroidal administration to improve the transfection efficiency and durability. The physical methods include electrotransfer (ET), and chemical methods include lipid and cationic polymer-based nanoparticles such as liposomes, as well as compacted DNA nanoparticles (DNPs). However, both the duration of treatment effect and the low efficiency of PR transduction remain key hurdles to overcome for nonviral retinal gene therapy.

Electrotransfer. ET, the application of a pulsed electric field to increase the permeability of cell membranes, is a 
physical method to improve gene delivery. Touchard et al. assessed ET as a nonviral gene delivery method to transfect the choroid and the retina without detaching the retina. ${ }^{34}$ The authors first injected pVAX1-LacZ encoding the $\beta$-galactosidase reporter gene suprachoroidally. After 7 days, only faint expression could be observed, highlighting the inefficiency of naked DNA to transduce retinal cells. They then developed an ET device, combining the administration of plasmid DNA into the SCS with the application of an electrical field, to enhance gene expression. With ET, efficient transduction of choroidal cells, RPE, and potentially PR was observed for at least 1-month when using a CMV promoter in adult rats. The procedure was noted to be devoid of side effects on the retina or the vascular integrity, recorded by angiographic, electroretinographic, and histological analyses. Furthermore, in a rat choroidal neovascularization $(\mathrm{CNV})$ model, a soluble vascular endothelial growth factor receptor-1 (sFlt-1)-encoding plasmid was injected into the SCS and ET was applied. In this study, the authors demonstrated that the area of induced $\mathrm{CNV}$ was significantly reduced, compared to the control.

Liposomal encapsulation. Liposomes are an attractive option for nonviral gene therapy. ${ }^{35}$ Liposomes, vesicles formed by phospholipid bilayers, are biocompatible and can be tailored for various sizes, release rates, hydrophilicity, and lipophilicity. ${ }^{36}$ Furthermore, the lipid bilayer of liposomes can integrate and pass through cell membranes without the need for electroporation. ${ }^{37}$ While liposomes containing genetic material have been evaluated for posterior eye diseases when applied topically, intravitreally, or subretinally, ${ }^{38}$ the application of liposomes delivered in the SCS is limited to a few preclinical applications. To evaluate the effect of extracellular matrix remodeling in the posterior sclera for myopia, Wan et al. injected liposomes containing the gene encoding tissue matrixmettalloproteinase-2 (TIMP2) suprachoroidally in a form-deprivation myopia model in guinea pigs. ${ }^{39}$ The authors noted that the levels of mRNA expression for collagen I and fibronectin were altered with the introduction of the TIMP-2 gene over 14 days. Nonviral gene delivery utilizing liposomes is primed for further research.

DNA nanoparticles. DNPs typically contain two key components, polyanionic DNA or RNA, and a cationic polymer chain. Poly-lysine, polyethylene glycol (PEG), and PEG-substituted poly-lysine have been used as cationic polymers to form DNPs. The complex of two oppositely charged components forms unique nanosized particles, typically within the range of $10-100 \mathrm{~nm}$ in diameter, which facilitate cellular uptake and intracellular trafficking of DNPs to the nucleus through nuclear pores. ${ }^{40}$ While cellular uptake of poly-lysine based DNPs typically uses clathrinmediated endocytosis, the PEG-substituted poly-lysinebased DNPs (20-25 nm) have been shown to use cell surface nucleolin receptor-mediated endocytosis. ${ }^{41,42}$ Chen et al. provided experimental evidence that a single plasmid DNA compacted with a 10-kDa PEG-substituted 30-mer lysine peptide (CK30PEG) binds to nucleolin receptors with nanomolar affinities and can be trafficked from cytoplasm to the nucleus by nucleolin-mediated process. ${ }^{42,43}$

A series of studies supported physical and chemical stability of DNPs under a range of physiologically relevant temperature, $\mathrm{pH}$, and salt concentration. Moreover, the shape of DNPs can be controlled-either rod or ellipsoid shape-by varying the poly-lysine counterion during the compaction process. This feature offers formulation options to develop customized nanoparticles for targeting different cell types. ${ }^{44}$ As noted above, another key feature of compacted DNPs is their payload capacity. While AAVs can contain plasmid sizes of up to $5 \mathrm{~kb}$, DNPs carry plasmids up to $20 \mathrm{~kb}$ and have been shown to be functional in vivo. ${ }^{45,46}$ Consequently, DNPs could become an excellent carrier of genes too large for AAV vectors, provided that their safety profile and long-term expression are proven.

Safety and efficacy of 30-mer lysine conjugated with 10kD PEG (CK30PEG)-based compacted DNPs following subretinal, ${ }^{32,47}$ intravitreal, and suprachoroidal administra$\operatorname{tion}^{48,49}$ have been successfully demonstrated. Han et al. successfully transfected RPE cells at a higher efficiency than naked DNA after subretinal administration without significant toxicity. ${ }^{46}$ Koirala et al. tested the ability of compacted DNPs to target the RPE, driving long-term, tissue-specific gene expression, and mediating proof-of-principle rescue in the rpe65(-l-) model of LCA. ${ }^{47}$ In this mouse model, DNPbased delivery of hRPE65 vectors resulted in persistent, therapeutically efficacious gene expression in the RPE. CK30PEG NPs demonstrated functional improvements of $\mathrm{RP}\left(\mathrm{Rds}^{+-}, \mathrm{Rho}^{-/}\right.$, and P23H models), Stargardt dystrophy in mouse models. Unlike commonly perceived shorter duration of transfection, DNP-based gene therapy resulted in the expression of reporter genes in RPE for up to 8-months.

Recently, we evaluated ocular tolerability and transfectability of DNPs in NHPs and rabbits. ${ }^{48}$ Suprachoroidal administration of DNPs was generally well tolerated in both rabbits and NHPs. Specifically, robust luciferase activity was observed in the retina and choroid of eyes that received suprachoroidal injections in NHPs and rabbits. In NHPs, the persistence of luciferase activity was observed through day 22 (the last terminal time point) with ellipsoid-shaped DNPs, while a decline was observed with rod-shaped DNPs on day 22. In rabbits, suprachoroidally injected DNPs (both rod and ellipsoid) and subretinal DNPs (rod shape) resulted in comparable luciferase activity. Long-term safety, effectiveness, and transfection durability of suprachoroidally delivered DNPs are currently under investigation.

\section{Future Directions}

The evolution of gene therapy has been remarkable over the last decade, and viral vector-based therapy has shown promising safety and efficacy in clinical trials. However, delivery methods require further study to reduce iatrogenic risks, and to potentially optimize targeting to the macular or peripheral retinal regions depending on the clinical need. For example, subretinal injections may be most effective in focally targeting PRs and RPE, and hence suitable for IRDs affecting the macula. Suprachoroidal injection could treat larger peripheral areas, typically affected by certain IRDs, such as RP or rod-cone dystrophies. This approach could potentially facilitate earlier treatment, before progression to the posterior pole, given the less invasive nature of the procedure. Intravitreal administration of gene therapy may potentially serve best for targeting retinal ganglion cells.

A potential challenge for suprachoroidal gene delivery by a microneedle is its limited regional specificity. Because the 
SC injection occurs at the pars plana, depending on the properties of the formulation and injection volume, the injectate does not specifically target the macula. Various strategies, such as the use of a "pushing" formulation after injecting a hydrogel formulation, the use of iontophoresis, the use of collagenase to expand the SCS, higher injection volume, or multiple injections, could be employed in future to optimize suprachoroidal delivery. ${ }^{50-52}$ Moreover, the use of catheter-based suprachoroidal delivery may facilitate gene transfer in the macula region.

Recent AAV technological advances are under preclinical investigation and are being considered for clinical development. This technology includes dual $\mathrm{AAV}^{53-55}$ and triple $\mathrm{AAV},{ }^{56}$ in which case, a large transgene is split into 2 or 3 separate vectors to ultimately reconstitute to a full-length gene, as well as intein-mediated protein trans-splicing, 57 which reconstitute large proteins from shorter precursor polypeptides (separately encoded by 2 or more independent AAV vectors). Although convincible preclinical data have been generated through subretinal administration, ${ }^{58}$ these platforms have not yet been assessed by the SC route. Future studies should focus on evaluating dual-vector and intein platforms through suprachoroidal delivery.

Suprachoroidal nonviral gene delivery could facilitate multi-treatment titration of therapy in response to the clinical outcomes and needs, as opposed to a single-time treatment using current viral vector-based techniques, which typically result in chronic therapeutic protein expression. To date, the most promising nonviral delivery platform, preclinically, seems to be the DNPs. As detailed above, these compacted nanoparticles hold a large cargo capacity, have been shown to be safe in humans after intranasal delivery, are efficacious in the mouse model of Stargardt's disease after subretinal administration, and have demonstrated luciferase activity after suprachoroidal delivery of luciferaseencoded plasmids containing DNPs in rabbits and NHPs. Further rigorous and long-term preclinical assessments of suprachoroidal DNP-based gene therapy are currently underway. Results from ongoing preclinical studies will determine the fate of nonviral retinal gene therapy.

\section{Concluding Remarks}

It is important to note that, while subretinal and intravitreal gene delivery have already been assessed clinically, suprachoroidal gene therapy is an emerging field, with limited preclinical evaluation to date. The safety, durability, and effectiveness of gene transfection, and gene expression following suprachoroidal gene delivery are yet to be proven in the clinic. Nonetheless, if successful, suprachoroidal gene therapy may pave the way for nonsurgical inoffice gene therapy. Furthermore, suprachoroidal nonviral gene delivery could lead to repeatable nonsurgical in-office gene therapy for disorders, such as Stargardt or Usher Syndrome, with transgenes greater than the carrying capacity of AAVs.

\section{Acknowledgments}

We thank Shelley Hancock, Director of Medical Affairs at Clearside Biomedical, Inc., for her assistance in designing and creating Fig. 1.

\section{Author Disclosure Statement}

Authors have an employment relationship with, and equity ownership in Clearside Biomedical. This work does not reflect any view or opinion of this corporation or management. All disclosures are in accordance with the policy of the Journal of Ocular Pharmacology and Therapeutics.

\section{Funding Information}

No funding was received for this article.

\section{References}

1. Takahashi, V.K.L., Takiuti J.T., Jauregui, R., et al. Gene therapy in inherited retinal degenerative diseases: a review. Ophthalmic Genet. 39, 560-568, 2018.

2. Dalkara, D., Kolstad K.D., Caporale, N., et al. Inner limiting membrane barriers to AAV-mediated retinal transduction from the vitreous. Mol. Ther. 17:2096-2102, 2009.

3. Food \& Drug Administration (internet). FDA approves novel gene therapy to treat patients with a rare form of inherited vision loss. Available at https://www.fda.gov/ news-events/press-announcements/fda-approves-novel-genetherapy-treat-patients-rare-form-inherited-vision-loss accessed February 2, 2020.

4. Moore N.A., Morral, N., Ciulla T.A., et al. Gene therapy for inherited retinal and optic nerve degenerations. Expert Opin. Biol. Ther. 18:37-49, 2018.

5. Seidman, C., and Kiss, S. Gene therapy: the next frontier for treatment of acquired and inherited ocular disorders. Retina Today. 69-71, 2015.

6. Moore, N.A., Bracha, P., Hussain, R.M., et al. Gene therapy for age-related macular degeneration. Expert Opin. Biol. Ther. 17:1235-1244, 2017.

7. Campochiaro, P.A., Nguyen, Q.D., Shah, S.M., et al. Adenoviral vector-delivered pigment epithelium-derived factor for neovascular age-related macular degeneration: results of a phase I clinical trial. Hum. Gene Ther. 17:167-176, 2006.

8. Askou, A.L., Alsing, S., Benckendorff, J.N.E., et al. Suppression of choroidal neovascularization by AAVbased dual-acting antiangiogenic gene therapy. Mol. Ther. Nucleids. 16:38-50, 2019.

9. Constable, I.J., Lai, C.M., Magno, A.L., et al. Gene therapy in neovascular age-related macular degeneration: threeyear follow up of a phase 1 randomized dose escalation trial. Am. J. Ophthalmol. 177:150-158, 2017.

10. Campochiaro, P.A., Lauer, A.K., Sohn, E.H., et al. Lentiviral vector gene transfer of endostatin/angiostatin for macular degeneration (GEM) study. Hum. Gene Ther. 28: 99-111, 2017.

11. Adverum Biotechnologies. Adverum Biotechnologies reports new interim data from cohorts 1 and 2 of OPTIC Phase 1 Trial of ADVM-022 intravitreal gene therapy for wet AMD at Angiogenesis, Exudation and Degeneration 2020 [Press Release], 2020. Available at http://investors .adverum.com/news-releases/news-release-details/adverumbiotechnologies-reports-new-interim-data-cohorts-1-and-2 accessed February 2, 2020.

12. REGENEXBIO. REGENEXBIO announces exclusive worldwide option and license agreement with Clearside Biomedical for evaluation of in-office delivery platform for RGX-314 [Press Release], 2019. Available at https://regenx bio.gcs-web.com/news-releases/news-release-details/regenx bio-announces-exclusive-worldwide-option-and-license accessed February 12, 2020. 
13. Touchard, E., Benard, R., Bigot, K., et al. Non-viral ocular gene therapy, pEYS606, for the treatment of non-infectious uveitis: pre-clinical evaluation of the medicinal product. J. Control. Release. 285:244-251, 2018.

14. Trapani, I., Puppo, A., and Auricchio, A. Vector platforms for gene therapy of inherited retinopathies. Prog. Retin. Eye Res. 43:108-128, 2014.

15. Cukras, C., Wiley, H.E., Jeffrey, B.G., et al. Retinal AAV8RS1 gene therapy for X-linked retinoschisis: initial findings from a phase I/IIa trial by intravitreal delivery. Mol. Ther. 26:2282-2294, 2018.

16. Yang, S., Wan, X., He, H., et al. Long-term outcomes of gene therapy for the treatment of Leber's hereditary optic neuropathy. EBioMedicine. 10:258-268, 2016.

17. Reichel, F.F., Peters, T., Wilhelm, B., et al. Humoral immune response after intravitreal but not after subretinal AAV8 in primates and patients. Invest. Ophthalmol. Vis. Sci. 59:1910-1915, 2018.

18. Dalkara, D., Bryne, L.C., Klimczak, R.R., et al. In vivodirected evolution of a new adeno-associated virus for therapeutic outer retinal gene delivery from the vitreous. Sci. Transl. Med. 5:189ra76, 2013.

19. Mowat, F.M., Gornik, K.R., Dinculescu, A., et al. Tyrosine capsid-mutant AAV vectors for gene delivery to the canine retina from a subretinal or intravitreal approach. Gene Ther. 21:96-105, 2014.

20. Yeh, S., Khurana, R.N., Shah, M., et al. Efficacy and safety of suprachoroidal CLS-TA for macular edema secondary to noninfectious uveitis: phase 3, randomized trial. Ophthalmology. 2020; DOI: 10.1016/j.ophtha.2020.01.006.

21. Peden, M.C., Min, J., Meyers, C., et al. Ab-Externo AAVmediated gene delivery to the suprachoroidal space using a 250-micron flexible microcatheter. PLoS One. 6:e17140, 2011.

22. Ding, K., Shen, J., Hafiz, Z., et al. AAV8-vectored suprachoroidal gene transfer produces widespread ocular transgene expression. J. Clin. Invest. 130:4901-4911, 2019.

23. Wykoff, C.C., Khurana, R.N., Lampen, S.I.R., et al. Suprachoroidal triamcinolone acetonide for diabetic macular edema: the HULK Trial. Ophthalmol. Retina. 2:874-877, 2018.

24. Campochiaro, P.A., Wykoff, C.C., Brown, D.M., et al. Suprachoroidal triamcinolone acetonide for retinal vein occlusion: results of the Tanzanite study. Ophthalmol. Retina. 2:320-328, 2018.

25. Chen, M., Li, X., Liu, J., et al. Safety and pharmacodynamics of suprachoroidal injection of triamcinolone acetonide as a controlled ocular drug disease model. J. Control. Release. 203:109-117, 2015.

26. Chiang, B., Jung, J.H., and Prausnitz, M.R. The suprachoroidal space as a route of administration to the posterior segment of the eye. Adv. Drug Deliv. Rev. 126:58-66, 2018.

27. Russell, S., Bennett, J., Wellman, J.A., et al. Efficacy and safety of voretigene neparvovec (AAV2-hRPE65v2) in patients with RPE65-mediated inherited retinal dystrophy: a randomised controlled, open label, phase 3 trial. Lancet. 390:849-860, 2017.

28. Sarin, H. Physiologic upper limits of pore size of different blood capillary types and another perspective on the dual pore theory of microvascular permeability. Vasc. Cell. 14: 2-14, 2010.

29. Martorana, G., Levine, M., Peden, M.C., et al. Comparison of suprachoroidal delivery via an Ab-Externo approach with the iTrack microcatheter versus vitrectomy and sub- retinal delivery for 3 different AAV serotypes for gene transfer to the retina. In: Poster presented at the Annual Meeting of the Association for Research in Vision and Ophthalmology; May 6-10, 2012; Fort Lauderdale, FL, USA. No. D748.

30. Woodard, K.T., Vance, M., Gilger, B., et al. Comparison of AAV Serotype 2 transduction by various delivery routes to the mouse eye. Mol. Ther. 24:S217-S218, 2016.

31. Yiu, G., Chung, S.H., Mollhoff, I., et al. Suprachoroidal and subretinal injections of AAV using transscleral microneedles for retinal gene delivery in nonhuman primates. Mol. Ther. Methods Clin. Dev. 16:179-191, 2020.

32. Han, Z., Conley, S.M., Makkia, R.S., et al. DNA nanoparticle-mediated ABCA4 delivery rescues Stargardt dystrophy in mice. J. Clin. Invest. 122:3221-3226, 2012.

33. Desrosiers, M., and Dalkara, D. Neutralizing antibodies against adeno-associated virus (AAV): measurement and influence on retinal gene delivery. Methods Mol. Biol. 1715:225-238, 2018.

34. Touchard, E., Berdugo, M., Bigey, P., et al. Suprachoroidal electrotransfer: a nonviral gene delivery method to transfect the choroid and the retina without detaching the retina. Mol. Ther. 20:1559-1570, 2012.

35. Takashima, Y., Tsuchiya, T., Igarashi, Y., et al. Noninvasive ophthalmic liposomes for nucleic acid delivery to posterior segment of eye. europepmc.org. Available at https://europepmc.org/abstract/med/23208042 (accessed August 1, 2019).

36. Santos, A., Altamirano-Vallejo, J.C., Navarro-Partida, J., et al. Breaking down the barrier: topical liposomes as nanocarriers for drug delivery into the posterior segment of the eyeball. Intechopen.Com. 2019. Available at https:// www.intechopen.com/online-first/breaking-down-the-barriertopical-liposomes-as-nanocarriers-for-drug-delivery-into-theposterior-seg (accessed August 5, 2019).

37. Kachi, S., Oshima, Y., Esumi, N., et al. Nonviral ocular gene transfer. Gene Ther. 12:843-851, 2005.

38. Masuda, I., Matsuo, T., Yasuda, T., et al. Gene transfer with liposomes to the intraocular tissues by different routes of administration. Invest. Ophthalmol. Vis. Sci. 37:19141920, 1996.

39. Wan L-Q, Liu G-X, Wang, L., et al. Dynamic change of collagen type I and fibronectin in posterior sclera of formdeprivation myopia after TIMP-2 gene transfect in guinea pig. Chinese J. Exp. Ophthalmol. 29:646-650, 2011.

40. Cai, X., Conley, S., and Naash, M. Nanoparticle applications in ocular gene therapy. Vision Res. 48:319-324, 2008.

41. Walsh, M., Tangney, M., O'Neill, M.J., et al. Evaluation of cellular uptake and gene transfer efficiency of pegylated poly-L-lysine compacted DNA: implications for cancer gene therapy. Mol. Pharm. 3:644-653, 2006.

42. Chen, X., and Davis, P. Compacted DNA nanoparticles transfect cells by binding to cell surface nucleolin. Mol. Ther. 13:S152, 2006.

43. Chen, X., and Davis, P. Intracellular trafficking of DNA nanoparticle and nucleolin antibody. Mol. Ther. 15:S196, 2007.

44. Farjo, R., Skaggs, J., Quiambao, A.B., et al. Efficient nonviral vectors ocular gene transfer with compacted DNA nanoparticles. PLoS One. 1:e38, 2006.

45. Fink, T.L., Klepcyk, P.J., Oette, S.M., et al. Plasmid size up to $20 \mathrm{kbp}$ does not limit effective in vivo lung gene transfer using compacted DNA nanoparticles. Gene Ther. 13:10481051, 2006.

46. Han Z., Koirala A., Makkia R., et al. Direct gene transfer with compacted DNA nanoparticles in retinal pigment ep- 
ithelial cells: expression, repeat delivery and lack of toxicity. Nanomedicine (Lond). 7:521-539, 2012.

47. Koirala, A., Makkia, R.S., Conley, S.M., et al. S/MARcontaining DNA nanoparticles promote persistent RPE gene expression and improvement in RPE65-associated LCA. Hum. Mol. Genet. 22:1632-1642, 2013.

48. Kansara, V., Yoo, J., Cooper, M.J., et al. Suprachoroidally delivered DNA nanoparticles transfect chorioretinal cells in non-human primates and rabbits. In: Poster presented at the Annual Meeting of the Association for Research in Vision and Ophthalmology; April 28-May 2, 2019; Vancouver, BC. No. A0096.

49. Taraborelli, D., Noronha, G., Moen, R., et al. A one-week study to evaluate safety, tolerability, and retinal cell transfection of non-viral vectors DNA nanoparticles administered by suprachoroidal injection. In: Poster presented at the Annual Meeting of the Association for Research in Vision and Ophthalmology; April 29-May 3, 2018; Honolulu, Hawaii. No. C0243.

50. Jung, J.H., Park, S., Chae, J.J., et al. Collagenase injection into the suprachoroidal space of the eye to expand drug delivery coverage and increase posterior drug targeting. Exp. Eye Res. 189:107824, 2019.

51. Jung, J.H., Desit, P., and Prauznitz, M.R. Targeted drug delivery in the suprachoroidal space by swollen hydrogel pushing. Invest. Ophthalmol. Vis. Sci. 59:2069-2079, 2018.

52. Jung, J.H., Chiang, B., Grossniklaus, H.E., et al. Ocular drug delivery targeted by iontophoresis in the suprachoroidal space using a microneedle. J. Control. Release. 277: 14-22, 2018.
53. Dyka, F.M., Boyle, S.L., and Chiodo, V.A. Dual adenoassociated virus vectors result in efficient in vitro and in vivo expression of an oversized gene, MYO7A. Hum. Gen. Ther. Methods. 25:166-177, 2014.

54. McClements, M.E., Barnard, A.R., Singh, M.S., et al. An AAV dual vector strategy ameriolates the Stargardt phenotype in adult Abca4 ${ }^{-1-}$ mice. Hum. Gene Ther. 30:590-600, 2019.

55. Carvalho, L.S., Turunen, H.T., Wassmer, S.J., et al. Evaluating efficiencies of dual AAV approaches for retinal targeting. Front. Neurosci. 11:503, 2017.

56. Maddalena, A., Tornabene, P., Tiberi, P., et al. Triple vectors expand AAV transfer capacity in the retina. Mol. Ther. 26:524-541, 2018.

57. Sarmiento, C., and Camarero, J.A. Biotechnological applications of protein splicing. Curr. Protein Pept. Sci. 20: 408-424, 2019.

58. Tornabene, P., Trapani, I., and Minopoli, R. Inteinmediated protein trans-splicing expands adeno-associated virus transfer capacity in the retina. Sci. Transl. Med. 11: eaav4523, 2019.

Received: October 29, 2019

Accepted: February 27, 2020

Address correspondence to: Dr. Thomas A. Ciulla Clearside Biomedical, Inc. 900 Northpoint Parkway Suite 200 Alpharetta, GA 30005

E-mail: thomas.ciulla@clearsidebio.com 